\title{
竞争-耐胁迫-杂草型植物对策理论及其应用研究进展
}

\author{
姚 忠, 吴永明, 游海林, 辛在军* \\ 江西省科学院微生物研究所,南昌 330096
}

\begin{abstract}
摘要: 竞争-耐胁迫-杂草型植物 ( CSR) 对策理论主要研究植物对环境胁迫和干扰的适应性特化。从功能生态学角度讲, CSR 理 论是通过解释各种功能性状协同变异的主导维度来反映植物的生态对策。而物种所采取的生态对策将决定其在群落中的生态 位, 并最终会影响生态系统过程与功能。自 1974 年 Grime 首次提出 CSR 对策的概念以来, CSR 对策思想就受到了生态学家很 大的关注,经过近半个世纪的发展, 目前已经形成了比较完整的理论和研究方法体系。试图在回顾 CSR 理论及其研究方法发 展的基础上, 阐述其基本内涵,并重点从功能性状变异空间、植物适应性、群落过程和生态系统特性等 4 个方面概述 CSR 理论 及其分析模型的验证及应用, 梳理其最新研究进展。CSR 理论已经得到了很多研究的广泛验证并应用于多种尺度上的植物生 态学研究,但仍有一些方面值得进一步发展完善,如第四区缺失问题、功能性状变异主轴以外的变异、CSR 对策与功能性状种内 变异和谱系关系的联系等方面需要深人研究。
\end{abstract}

关键词: 竞争-耐胁迫-杂草型植物 (CSR) 对策理论; 生态对策; 适应性特化; 干扰;胁迫; 竞争

\section{Research progress of plant competitor-stress tolerator-ruderal (CSR) theory and its application}

\author{
YAO Zhong, WU Yongming, YOU Hailin, XIN Zaijun * \\ Institute of Microbiology, Jiangxi Academy of Sciences, Nanchang 330096, China
}

\begin{abstract}
Competitor, stress-tolerator, ruderal (CSR) theory, regarded as one of the best developed plant ecological strategy schemes, mainly studies the adaptive specialisation that plants evolve to achieve fitness in response to natural selection pressures, resulting in a triangular distribution of species optima along axes of habitat productivity and disturbance. From the perspective of functional ecology, the CSR theory reflects plant ecological strategies by explaining the principal functional spectra in the multidimensional trait space, that is, the world-wide economics spectrum and the size spectrum as fundamental gradients of plant evolution are major components of the CSR strategy variation. The ecological strategies adopted by plants then determine their ecological niche in the community and ultimately affect ecosystem processes and functions. Thus the CSR theory could not only be used as a framework for functionally classifying plants and predicting impacts of environmental change on vegetation, but also serves as a conceptual framework for investigating and interpreting community processes and ecosystem functioning. Since the concept of CSR strategies was first raised by Grime in 1974, the idea of CSR strategies has attracted much attention from ecologists, and the CSR theory has formed a relatively complete system of theories and research methods over the last half century. In this paper, we introduce the development and characteristics of the CSR theory and its methods to expound the core philosophy of the theory, review the verification and application of the CSR theory and its models by addressing the relevance of CSR strategies to functional trait variations,
\end{abstract}

基金项目: 国家自然科学基金项目 (31860119); 江西省重点研发计划项目 (20181BBG70036, 20202BBG73006); 江西省青年人才项目 (20204BCJL23040)

收稿日期: 2021-01-06; 网络出版日期:2021-08- 17

*通讯作者 Corresponding author.E-mail: zaijunxin@126.com 
species adaptation, community processes and ecosystem properties, and summarize the advances in this theory. The CSR theory has been extensively validated by many studies and widely applied as a very useful analysis tool to plant ecology research on a variety of scales, but it needs further research, such as the missing fourth corner in the Grime's C-S-R triangular model, the rest functional variations beyond the principally functional trait spectra that are not explained by the CSR scheme, and the relationships between CSR strategies and the intraspecific trait variation and phylogenetic signal. Especially for the rest functional variations, the CSR theory does not preclude the existence of additional axes of variability subordinate to the principal functional spectra, nor that these may be certainly key to fitness and survival in specific cases. The CSR theory could be seen as a general adaptive strategy, since it is consistent with economics and size tradeoffs evident throughout the tree of life, hinting at overarching natural selection filters operating universally within the biosphere.

Key Words : competitor-stress tolerator-ruderal ( CSR) theory; ecological strategy; adaptive specialisation; disturbance; stress; competition

自 1974 年 Grime 首次提出 CSR 对策的概念以来, CSR 对策思想就受到了生态学家很大的关注 ${ }^{[1-2]}$ 。特 别是在当前全球变化和生态保护研究的强烈需求背景下,有越来越多的研究关注气候变化或土地利用变化对 物种分布、群落构建和生态系统功能的影响 ${ }^{[3-4]}$ 。CSR 对策反映了植物在一定生境生产力和干扰水平下的适 应性响应 ${ }^{[5]}$, 是植物与环境相互作用的结果 ${ }^{[6]}$ 。从功能生态学角度讲, CSR 理论是通过解释各种功能性状协 同变异的主导维度来反映植物的生态对策 ${ }^{\left[{ }^{3]}\right.}$ 。而物种所采取的生态对策将决定其在群落中的生态位, 并最 终会影响生态系统过程与功能 ${ }^{[7-8]}$ 。因此, CSR 理论及其模型不仅可用于植物功能性分类和预测环境变化对 植被的影响, 也可以作为一个概念性框架进行比较植物生态学研究, 从而实现在共同的基础上评估环境因素 或管理方式对生物多样性、生态系统稳定性等的影响。

CSR 理论经过近半个世纪的发展,已经形成比较完整的理论和方法体系。由于其理论假设简明、分析方 法实用, 目前已作为一个实用的生态学工具被广泛应用于物种适应性、群落过程和生态系统特性等相关研究 中, 但国内相关研究较少。本文在简要回顾 CSR 理论及其分析模型发展的基础上, 梳理其在植物生态学研究 中的应用进展, 并探讨当前研究的不足之处以及未来发展的方向, 以供国内相关研究者参考。

\section{CSR 理论及其分析模型的发展}

\subsection{CSR 理论的形成及其基本内涵}

1974 年 Grime 在《Nature》发表了《基于对策的植被分类》, 首次提出 CSR 对策思想 ${ }^{[9]}$ 。1979 年 Grime 出 版《植物对策与植被过程》, 初步论述了 CSR 理论 ${ }^{[5]}$ 。2001 年 Grime 出版该书的第二版《植物对策、植被过程 和生态系统特性》 ${ }^{[10]}$ 。2012 年 Grime 和 Pierce 共同出版了《塑造生态系统的进化对策》, 提出了普适性适应 对策观点, 将植物对策理论进一步延伸到包括对微生物、动物在内的各种生物适应对策分析 ${ }^{[8]}$ 。因此, CSR 理论是由 Grime 及其团队共同建立的关于植物生态对策的理论,其具有以下 4 方面基本内涵:

(1) CSR 理论假设胁迫和干扰是影响植物适合度的两个主要环境因素。Grime ${ }^{[11]}$ 认为, 胁迫是限制植物 干物质增长的环境因素 (如养分不足), 而干扰是破坏植物生物量积累的外力 (如放牧), 将胁迫和干扰水平进 行不同组合可以形成适合植物生长的 3 种基本生境类型,但植物不能长期生存在同时受高度胁迫和干扰作用 的极端生境中 ( 图 1)。

(2) CSR 理论所建立的植物对策类型谱包括 3 种基本对策和 16 种次级对策 (图 1)。3 种基本生境类型 对应竞争型植物 $\mathrm{C}$ ( Competitor)、耐胁迫型植物 S (Stress-tolerator) 和杂草型植物 R(Ruderal) 这 3 种基本对策 型植物, 占据三角模型 (triangular model) 的 3 个角, 是植物对极端环境的适应性特化; 而适应于不同水平的胁 迫和干扰之间的特定平衡, 植物进化出多种次级对策占据三角形的中间区域, 如竞争型杂草类植物 ( CR 对策 者) 等 ${ }^{[12]}$ 。 

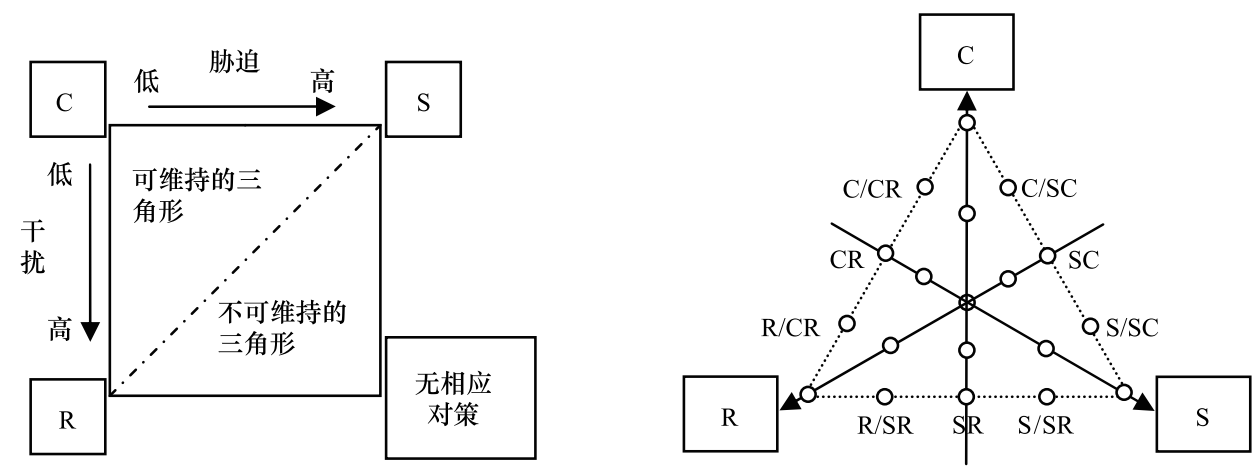

图 1 植物 CSR 对策的基本分类体系

Fig.1 The classification of plant CSR strategies

C, 竞争型植物 competitor; S, 耐胁迫型植物 stress-tolerator; R, 杂草型植物 ruderal. (a) CSR 理论假设的植物沿生境生产力和干扰梯度的生 态位分布 (仿自 Grime ${ }^{[5]}$ ); (b) CSR 分类空间中的 19 种对策类型(仿自 Hodgson 等 ${ }^{[11]}$ ), 以小空心圆表示

(3)植物的 CSR 对策表现是物种在不同功能间权衡资源配置的结果。植物必需权衡有限资源在提高竞 争能力、耐胁迫能力和抗干扰能力之间的投资分配问题, 而权衡的结果是在一种情形下能增加物种适合度的 性状组合将不可避免地降低其在另外情形下的适合度,如在贫痊生境中植物主要表现为增强耐胁迫性而降低 其对养分的竞争能力 ${ }^{[8]}$ 。

(4) CSR 理论主要研究植物在定居阶段的适应性特化。很多研究发现, 植物在定居阶段和繁殖更新阶段 的性状间存在弱相关性 ${ }^{[2]}$,但各阶段的性状变异是高度集中的 ${ }^{[13-14]}$ 。

\subsection{CSR 分析模型的发展}

最初 Grime 提出 CSR 对策概念时, 对 C 对策者的界定是基于冠层高、侧向扩展和枯落物积累量的综合形 态指数, 而用最大相对生长率界定 $\mathrm{S}$ 对策者, 但对 $\mathrm{R}$ 对策者没有明确界定 ${ }^{[9]}$ 。之后 Grime 又利用形态、生理和 繁殖等 20 种特征建立了二叉式检索表来判别植物的 CSR 对策类型, 但这需要通过费时费力地野外调查和实 验室分析来采集足够的数据。近些年来功能生态学的发展, 为 CSR 三角模型的定量分析提供了新的思考 角度。

\subsubsection{Hodgson 等的模型}

1999 年, Hodgson 等 ${ }^{[12]}$ 基于冠层高、叶干物质含量、开花时间、花期、侧向扩展、叶干重和比叶面积 7 种功 能性状与竞争性、耐胁迫性和杂草性的相关性,实现了对植物 CSR 对策类型的界定。通过该模型与 Grime 的 二叉式检索表对英国 493 种草本植物的 CSR 对策类型分析, 发现两种方法的分类结果一致性达到了 $96 \%$ 。

Hodgson 等建立的 CSR 分析方法首次将 CSR 理论转化为一种实用的生态学方法,由于该模型简单,且创 建了定制表格工具, 因此迅速从英国应用到全球各地的生态学研究中,也进一步扩大了 CSR 理论的影响 力 $^{[15-16]}$ 。但该模型至少存在 4 方面问题: (1) 用于分析的性状有定类变量,如花期、开花时间等; (2) 模型中 开花时间不能用于比较寒带、温带和热带植物, 也不能严格地应用到南半球; (3) 由于要利用冠层高, 该模型 只适用于草本植物而没有涵盖木本植物; (4) 对一些特殊植物的 CSR 类型界定可能存在问题, 如肉质植物、盐 生植物、以茎为主要光合作用器官的植物等。

\subsubsection{Pierce 等的模型}

针对 Hodgson 等的模型存在的问题, Pierce 等 ${ }^{[17]}$ 利用叶干物质含量、比叶面积和叶面积 3 种叶性状与多 种功能性状的变异主轴进行回归分析, 从而建立了与 CSR 理论相对应的生态对策分析模型。2017 年 Pierce 等利用收集于 6 个大陆的 3068 种植物的这 3 种叶性状值建立了全球校正的 CSR 分类系统 ${ }^{[3]}$, 并形成了电子 表格工具“StrateFy”,从而实现了在一个通用框架下对不同地理区域的植物 CSR 对策进行分析比较。

相对于 Hodgson 等的模型,Pierce 等建立的 CSR 分析模型虽仍对一些特殊植物的 CSR 类型界定存在问 
题, 但表现出 3 个方面的突出优点: (1) 选用的都为数量性状, 不仅直观、易测定, 而且适用于各种植物类群; (2) 可应用于一般的维管束植物,包括木本、草本、蕨类和水生植物, 同时适用于群落内物种和种群内个体比 较; (3) 基于功能生态学理论, 利用主成分分析方法 (PCA) 实现将功能性状变异空间转化为与 CSR 理论相对 应的三角形排序空间。因此,Pierce 等建立的 CSR 模型同时具有理论和实际应用价值。

\section{CSR 理论及其模型的验证及应用}

CSR 对策思想自提出以来,虽受到一些学者的质疑,但 CSR 理论及其分析模型的有效性就在于其简单明 确的假设和客观的多变量分析方法 ${ }^{[18-20]}$ 。目前 CSR 理论及其模型已经被广泛应用于物种适应性、群落过程 和生态系统特性等相关的理论和实践研究中, 同时这些研究也为 CSR 理论提供了实证支持和扩展补充。

2.1 功能性状的变异空间

由于没有预先的比较基准, 事实上很难对 CSR 分析模型的有效性作出定量估计,而基于功能生态学对性 状变异进行多变量分析正试图提供这样一个基线, CSR 理论也是首个提出可以通过性状空间降维进行植物生 态对策分析的理论。

很多研究发现, 植物功能性状的变异主轴可以概括为经济型谱 (economics spectrum) 和大小谱 ( size spectrum)。如 Laughlin 等 ${ }^{[21]}$ 对北美 133 种植物的 10 种功能性状、Díaz 等 ${ }^{[1]}$ 对收集于全球 46085 种植物的 6 种性状的研究; 在群落水平上, Douma 等 ${ }^{[22]}$ 对分布于荷兰多种生境的 7644 个植被样方的 12 种功能性状的研 究,都发现类似的性状变异格局。即使是对功能性状数据采用不同的转换方法,也不会改变经济型谱与大小 谱在性状变异空间中的正交关系 ${ }^{[15,23]}$ 。可见, 功能性状变异的这两条主轴是广泛存在的, 是植物功能权衡的 高度代表, 并在很大程度上独立于地理梯度、植物区系、系统发育群或生活型。其中, 经济型谱是一条有关植 物资源利用对策的特化轴 ${ }^{[24]}$, 大小谱反映植物对优势度的控制, 而其它营养性状和繁殖性状等都可以协同变 化而成为这两个主轴的组分 ${ }^{[22,25]}$ 。Díaz 等 ${ }^{[1]}$ 认为, 功能性状间的权衡决定了植物的生态对策。从功能生态 学角度讲, CSR 理论正是通过最大可能地解释各种功能性状的协同变异来反映植物的生态对策 ${ }^{[1,3]}$ 。因此通 过客观的多变量分析方法如 PCA 确定的性状变异轴与 CSR 对策类型的变异轴应具有高度一致性 ${ }^{[23]}$ 。 Cerabolini 等 ${ }^{[15]}$ 利用 PCA 对分布于欧洲 506 种植物的 11 种功能性状进行分析, 发现性状变异主轴与物种的 CSR 对策类型呈显著相关性, 第一主成分与竞争型策略组分显著正相关而与杂草型和耐胁迫型策略组分负相 关, 第二主成分与耐胁迫型策略组分正相关而与杂草型策略组分负相关, 很好地验证了 CSR 对策可以反映植 物功能性状之间的权衡决定的适应性特化。

叶经济型谱和叶大小谱变异也是普遍存在的 ${ }^{[23]}$, 而且各种叶性状间的权衡与根、茎、花以及植株整体性 状间的权衡都存在密切相关 ${ }^{[1,26-27]}$ 。对来自全球 371 种植物的研究发现, 分别由 14 种性状与叶干物质含量、 比叶面积和叶面积这 3 种叶性状组成的两种变异空间之间存在明显的一致性 ${ }^{[3]}$ 。因此, 可以合理地假设由这 3 种叶性状描述的多变量功能空间就能代表植物整体的功能变异, 从而支持 Pierce 等建立的 CSR 模型。

$2.2 \mathrm{CSR}$ 对策与植物适应性的关系

CSR 理论认为植物适应性的差异可由生境生产力和干扰予以解释和预测, CSR 对策是反映植物对各种 环境条件综合适应的一个妥协策略 ${ }^{[8,28]}$ 。

\subsection{1 植物的资源利用对策}

Grime 认为 ${ }^{[14]}$, 植物对生境生产力响应的差异主要表现在 CSR 体系中的 C-S 轴上, 反映了植物在资源竞 争与耐胁迫性之间的权衡, 但不存在同时具有高或低的竞争力和耐胁迫能力的物种。植物资源利用对策的这 种权衡可以对应于叶片的“快-慢” 经济型谱 ${ }^{[29-30] 。}$

生境生产力决定了植物采取特定的 CSR 对策类型。在肥沃生境中 C 对策者是优势种 ${ }^{[31]}$, 但在养分脉冲 式变化的生境中 $S$ 对策者将成为优势种 ${ }^{[32]}$, 而在长期贫痊的生境中植物将表现出典型的耐胁迫性 ${ }^{[13]}$ 。如贫 痊土壤作为砾质土草地和砂质土草地共同的主要环境篮, 导致这两类草地都以耐胁迫性植物为优势种 ${ }^{[33]}$ 。 
水分梯度强烈影响湿地植物生态对策的分布格局。如对斯洛文尼亚一个湿地的研究发现,具有 $\mathrm{C}$ 和 $\mathrm{CR}$ 对策 的植物比例与湿度负相关而与高程正相关, CS 对策者比例与湿度正相关而与高程负相关 ${ }^{[34]}$ 。而在较大尺度 上,气候条件可能是反映环境胁迫的关键指标, 如降水对黄土高原旱作农业区农田杂草 ${ }^{[35]}$ 、气温对巴西南部 亚热带森林 ${ }^{[36]}$ 等形成独特的 CSR 对策类型具有重要影响。在多种环境胁迫下植被将形成多样化的生态对 策。如林下植物针对光合有效辐射和土壤水分的不同胁迫水平采取了不同的生态对策, 受中低程度土壤水分 和光照胁迫的生境更适于 $\mathrm{C}$ 对策者, 中等光胁迫强度而土壤水分充足的生境更适于中间型的 CSR 对策者, 而 高光和中等干旱的生境更适于 CS 对策者 ${ }^{[37]}$ 。但不同植物即使具有相近的 CSR 对策类型也可能以不同方式 响应环境胁迫。如对 4 种海岸沙丘植物的研究发现, 这些植物虽都表现为 $\mathrm{R} / \mathrm{CR}$ 或 $\mathrm{C} / \mathrm{CR}$ 对策,但它们或通 过形成非腺性毛状体以避免高温和干旱胁迫, 或通过产生次生代谢物以应对胁迫 ${ }^{[38]}$ 。

2.2.2 植物的抗干扰对策

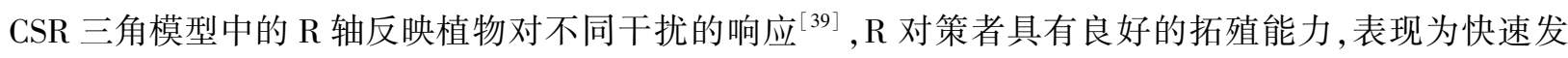
芽、短的生活周期、开花早和繁殖投人大等特征, 这类植物在受干扰的裸地生长良好, 但随着干扰减少生境中 的这类植物也将减少 ${ }^{[5]}$ 。

CSR 对策可以反映植物对干扰的响应。在高放牧压力下植物主要通过表现出 $\mathrm{R}$ 对策以躲避牧食, 而不 是形成高成本的防御性结构或不利适口性的次生代谢物 ${ }^{[40]}$ 。适度放牧将压制优势种而增加机会种。如对阿 尔卑斯山草地的研究发现,相对于未放牧的单优势种天然草地,受放牧干扰的草地有 5 个共优种,具有更多的 从属种和 CSR 对策类型; 且放牧草地上出现了较多的 R 对策者和特有的 CR 对策者, 说明畜肥改善了高山贫 痊的生境条件 ${ }^{[41]}$ 。但畜肥过高将不利于生物多样性。如在低畜肥而高放牧强度的草地, CSR 对策的丰富度 和均匀度都要高于高畜肥而低放牧强度的草地, 而且 CSR 对策的均匀度要比物种均匀度的变异更显著, 表明 CSR 对策均匀度可以作为评价群落利用不同生态位能力的更直接测度 ${ }^{[42]}$ 。相对于放牧, 刈割不仅可以选择 性地去除大量高的草本植物而促进杂草型植物生长 ${ }^{[43]}$, 而且割草引起的土壤养分流失也将有利于耐胁迫型 植物生长 ${ }^{[44]}$, 表明割草通过显著降低生物量生产和物种的竞争性而为弱竞争者提供生长机会。物种响应干 扰的 CSR 对策可以反映其受损害程度。如对北美阿巴拉契亚山脉的冰雪风暴灾害研究发现, C 对策者遭受 的损害最为严重, 特别是枝条折断, 但是这类树种能通过快速重建冠层而抵抗突发的灾害事件, 因而死亡率较 低和灾后恢复力较强; $\mathrm{S}$ 对策者受损害程度最小,而 $\mathrm{R}$ 对策者与冰雪风暴损害的相关性不明显 ${ }^{[45]}$ 。 $\mathrm{Grime}^{[10]}$ 也认为, 具有 $\mathrm{C}$ 对策的草本植物能通过快速重建高而稠密的株丛而幸存于不频发但严重的破坏事件, 因而具 有较强的表型可塑性和灾后恢复力。

近几十年来由于传统农林系统的变化导致全球范围的抛荒和停止放牧等现象,对生物多样性造成了不利 影响 ${ }^{[46-47]}$ 。如对丹麦半自然草地的研究发现, 随着传统的自由放牧转变为夏季放牧,一些 C 对策者的盖度 增加, 但更多具有 $\mathrm{S}$ 对策的低矮草本植物的盖度减少, 表明夏季放牧方式不足以有效保护草地植物多样 性 ${ }^{[48]}$ 。对英国休耕地植被的调查也发现, 随着休耕年限增加, 植被的竞争型对策组分值增加而杂草型对策组 分值减小 ${ }^{[49]}$, 证实了 Grime 的土地抛荒将促进竞争型植物出现的观点 ${ }^{[10]}$ 。外来物种人侵被认为是导致生态 系统变化的最重要因素之一 ${ }^{[50-51]}$ 。很多研究都发现, 外来植物和本地植物的生态对策存在明显不同, 外来人 侵种主要表现出 $\mathrm{R}$ 和 $\mathrm{C}$ 选择, 而 $\mathrm{S}$ 选择相对较少 ${ }^{[52-53]}$, 表明外来种成功人侵与其自身具有较强的资源竞争 力和抗干扰能力密切相关。因此, CSR 对策分析有助于辨识具有较高人侵性的外来种, 以更好地保护生物多 样性。

\subsection{CSR 对策与群落过程的关系}

Grime 在其专著《植物对策、植被过程和生态系统特性》中, 重点从优势性、群落构建、物种珍稀性和灭绝、 拓殖和人侵、演替、物种共存 6 个方面论述了植物对策与群落过程之间的相关性 ${ }^{[10]}$ 。很多研究也表明 CSR 对 策与植被动态性之间存在可预测的相关性, Wilson 等认为 CSR 理论是目前群落生态学中最具综合性和系统 性的一个理论 ${ }^{[54]}$ 。 


\subsubsection{CSR 对策与群落构建}

群落构建一直是植物生态学研究的热点论题 ${ }^{[55-56]}$ 。很多研究表明,基于中性理论的扩散限制、基于生态 位理论的环境滤篎和竞争排斥等多个过程共同影响群落构建 ${ }^{[57-58]}$, 但目前对群落构建机制尚无统一的认 识 ${ }^{[59]}$ 。群落构建的不同驱动力并不是相互对立的, 它们可能对不同性状或在不同空间尺度上发挥作 用 ${ }^{[60-62]}$ 。CSR 对策反映植物各种功能性状协同变异的主轴, 可以为理解群落构建提供一个总体框架。为此, Grime 等 ${ }^{[8]}$ 提出了群落构建的双重滤笁模型 (twin-filter model), 认为第一级滤笁与 CSR 对策有关 ( 又称为 CSR 篮), 促使进人该群落的物种关键性状趋同; 第二级滤笁主要与持久力、存活力、繁殖更新等性状相关而 与 CSR 性状不直接相关,将导致共存物种的这些性状趋异。Pierce 等 ${ }^{[27]}$ 对意大利 371 种植物的 15 种性状进 行 PCA 分析发现, 前两个主成分分别代表经济型谱和大小谱,但第二主成分还与种子性状存在弱相关,第三 主成分则与种子性状存在显著相关性而形成一个种子权衡维,表明除了 CSR 对策代表的基本轴外,还有未被 包含的性状变异存在。这与双重滤篮模型的假设是一致的, 说明植物在 CSR 三角形内进行各种关键功能之 间的权衡而形成基本对策, 同时可能也需要将基本对策与局域环境特殊性间的关系予以调和, 因而将植物的 生态对策归并为 CSR 对策所产生的局限性并不妨碍进一步生态细化,特别是为应对局域环境的特异性而形 成独特的繁殖对策、物候对策或共生对策等 ${ }^{[63-65]}$ 。

CSR 对策分析可以为洞察群落构建提供一个直接联系。对阿尔卑斯山的放牧草地和自然草地的对比研 究发现,在同一类型草地内植物主要表现出响应放牧有无的 R 或 S 对策,但不同类型的草地植物都主要表现 出响应高山胁迫环境的 S 选择,表明环境篮将导致共存物种在总体 CSR 对策类型上趋同,而放牧将促使局域 植物的 CSR 对策类型多样化和生态位分化 ${ }^{[41]}$ 。Grime 认为 ${ }^{[66]}$, 干扰是局域尺度上性状分异和物种共存的重 要驱动力, 而生境生产力引起的性状趋同可同时作用在群落和生态系统水平上。但沿着资源可利用性梯度可 能不一定存在功能变化的一致性趋势。如在极端高或低生境生产力下,群落表现出 $\mathrm{C}$ 或 $\mathrm{R}$ 的趋同对策,而在 中等生境生产力下群落则表现出许多次级对策, 表明生物和非生物制约的释放而允许多种生态对策的共 存 ${ }^{[67]}$ 。可见, 群落构建是多种生态滤篮综合作用的过程, CSR 对策分析可以为研究和理解群落构建提供一个 强有力的生态学工具。

\subsubsection{CSR 对策与群落演替}

演替是动态的群落构建, 目前对于群落结构是确定性的还是历史偶然性的观点虽仍有争议 ${ }^{[68]}$, 但很多研 究表明, 演替过程中物种组成往往是不可预测的, 但功能性变化是确定性的 ${ }^{[69-70]}$ 。因此, 群落的功能性变化 分析可能更有助于反映演替过程及其驱动力。

原生演替发生在从未有过任何生物的裸地。对冰川前陆的原生演替研究发现, 早期由于雪崩、冰川融水 等干扰, 先锋植物以 $\mathrm{R}$ 对策者为优势种,而在演替中后期则以 $\mathrm{S}$ 对策者为优势种,表明在高山严酷环境中,相 对于竞争,有效的耐胁迫能力和资源保存与利用能力显得更为重要 ${ }^{[16]}$ 。但演替过程中新出现的干扰会影响 这种轨迹。如对阿尔卑斯山岩屑区的原生演替研究发现, 群落对策从早期的 R 选择到后期的 $\mathrm{S}$ 选择, 但在放 牧影响下, 后期群落又进一步特化形成多种生态对策,包括 $\mathrm{C} / \mathrm{CR} 、 \mathrm{R} / \mathrm{CR}$ 和 $\mathrm{CR} / \mathrm{CSR}$, 表明食草动物在抑制或 加速演替进程中有重要作用 ${ }^{[3]}$ 。与资源贫痊的高山环境相反, 受火山泥流干扰的生境具有一定养分, 因此在 其演替过程中, 群落功能性的主要变化虽然仍是 $\mathrm{R}$ 对策组分值不断降低而 $\mathrm{S}$ 对策组分值逐渐增加, 但 $\mathrm{C}$ 对策

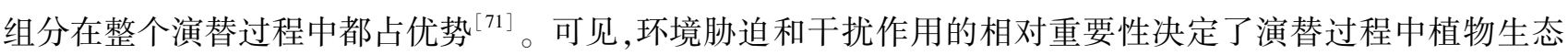
对策的变化格局。

与原生演替不同, 次生演替发生在原有植被人为破坏后的次生裸地。Grime ${ }^{[11]}$ 认为, 次生演替早期以 $\mathrm{R}$ 对策者而在后期则以 $\mathrm{S}$ 对策者为优势种, 而 $\mathrm{C}$ 对策的重要性取决于生境生产力。对苜蕧农牧地抛荒的研究 发现类似演替轨迹, 但竞争性由于群落优势种一直是竞争力强的多年生植物而变化不大 ${ }^{[72]}$ 。与抛荒牧地不 同, 抛荒耕地由于农作残留肥力因而物种竞争较激烈, 但群落的耐胁迫性变化不明显, 表明土地利用历史对演 替有重要影响 ${ }^{[73]}$ 。但较高的农作残留肥力不利于 $\mathrm{S}$ 对策者, 如抑制了英国低地草地的耐胁迫型植物生 
长 ${ }^{[74]}$ 。也有研究发现, 次生演替早期的优势种不一定具有 $\mathrm{R}$ 对策。如对延河流域森林草原区的植被恢复研 究发现, SR 对策者在整个恢复期都占据优势地位并呈增多趋势, 而 C 和 SC 对策者则均逐渐减少,这可能是 因为土壤养分含量虽总体呈上升趋势, 但植物的生存环境并未改善到不存在胁迫的程度 ${ }^{[75]}$ 。对牧地抛荒后 自然成林的研究也发现,植被呈现出草本群落-灌木群落-乔木群落的演替过程,群落总体的生态对策也相应 地发生了 S-SC-C/SC 的变化 ${ }^{[76]}$ 。因此, 群落演替轨迹可能不存在一致的模式, 而 CSR 对策分析可以指示演 替阶段和方向而无需考虑具体的物种组成,因此有助于探究演替过程中的群落构建机制。

2.4 CSR 对策与生态系统特性的关系

CSR 对策反映了植物各种功能性状协同变异的主导维度, 而植物的功能性状可与生态系统过程与功能联 系起来, 如叶调落物分解是地球化学循环中的一个重要过程 ${ }^{[77]}$ 。Grime 在专著《植物对策、植被过程和生态 系统特性》中, 重点从营养结构、生产力和稳定性等 3 个方面论述了植物生态对策与生态系统特性之间的 联系 ${ }^{[10]}$ 。

调落物分解是陆地生态系统碳和养分循环的关键过程 ${ }^{[78-79]}$ 。研究发现, 耐胁迫性高的植物由于产生较 多的支持组织,其调落物分解较慢, 从而影响养分循环、土壤形成等 ${ }^{[25]}$ 。这与 Reich $^{[29]}$ 提出的 “慢” 对策物种 与 “慢” 的生态系统过程相联系的观点一致, 即具有较高组织密度、较长组织生活周期的资源保守性物种 (即 $\mathrm{S}$ 对策者), 其调落物分解也比较慢。调落物的分解速率影响碳周转。对分布于全球 818 种植物的叶性状与其 调落物分解的相关性研究发现,资源保守性物种将获得较 “慢”的碳投资回报,表现出长寿命、较高的比叶重 和较低的叶氮含量, 这种关联性为植物整体碳策略和生物地球化学循环之间建立了一个新的联系 ${ }^{[80]}$ 。事实 上, 生态对策可以在一定程度上直接反映植物自身的碳分配格局。如对湿地植物的研究发现, 相比 $\mathrm{C}$ 和 $\mathrm{R}$ 对 策者, $\mathrm{S}$ 对策者具有较高的结构性成分 (如木质素), 而 $\mathrm{C}$ 和 $\mathrm{S}$ 对策者比 $\mathrm{R}$ 对策者具有更高的存储性成分 (如 淀粉), 且具有不同生态对策的植物其碳水化合物分配受生境养分含量变化的影响也不同, 表明生态对策和 生境养分水平可能是植物碳分配的关键驱动因素 ${ }^{[81]}$ 。因此, 探索调落物可分解性与植物 CSR 对策之间的联 系,有助于深人理解植被-土壤反馈机理和改进全球碳循环模型。

植被净初级生产力 (NPP) 对理解碳循环及其对气候变化的响应是至关重要的 ${ }^{[82]}$ 。CSR 理论认为, NPP 与生物量之间存在 $\mathrm{S}$ 形关系, 曲线较高的渐近线部分对应于 $\mathrm{C}$ 对策者,较低的渐近线部分对应于 $\mathrm{S}$ 或 $\mathrm{R}$ 对策 者, 而次级对策者对应于曲线的陡峭部分 ${ }^{[8]}$ 。Jenkins 等 ${ }^{[83]}$ 对收集于全球 709 条植被 NPP 与生物量的成对数 据进行分析, 发现相对于基于生态学代谢理论的线性模型和非对称性竞争假说的二次曲线模型, 基于 CSR 理 论的 S 形曲线模型能更好地预测 NPP 与生物量的异速比例关系, 表明植物的生态对策及其生长环境通过级 联作用向上调节 NPP, 因此可以基于碳循环与植物生态对策之间的联系来解释和预测全球生物量生产和碳 循环格局。生物多样性一直被认为对生物量生产有正效应 ${ }^{[84-85]}$, 虽然目前对两者关系仍没有统一模式 ${ }^{[86]}$, 但 Grime ${ }^{[87]}$ 提出的 “驼背” 模型受到了广泛关注。这种模式在对意大利北部草地的研究中得到了很好地印 证, 并发现中等生物量的群落具有最高的物种丰富度和 CSR 对策类型多样性; 而在极端低或高生物量的群落 中, 物种丰富度较低, 并分别以 $\mathrm{R}$ 和 $\mathrm{C}$ 对策者为优势种, 表明在极端生物量情况下植物将为生存而表现出高 度的适应性特化 ${ }^{[67]}$ 。Kelemen 等 ${ }^{[88]}$ 对匈牙利大平原的研究也发现类似格局, 但低生物量群落是以 $\mathrm{S}$ 对策者 为优势种, 这是因为这类群落受土壤湿度的高季节性变异和土壤高碱度的共同胁迫影响。因此, 植物的 CSR 对策分析有助于深人理解生物量-生物多样性关系。

植被是地表能量收支过程中的基本参量 ${ }^{[80-90]}$ 。对森林热力学效率的研究发现, 相比具有较高杂草型对 策组分值的幼林或人工林, 天然成熟林具有较高比例的竞争型和耐胁迫型对策组分, 不仅生物量存储高, 而且 能更有效地耗散表面温度, 表明 CSR 模型可与生态系统的热力学动态相关联来指示生态系统的复杂性 ${ }^{[91]}$ 。 CSR 理论认为植被抵抗力与耐胁迫型植物、植被恢复力与杂草型植物和竞争型植物的特性间存在可预测的关 系, 因而可预测植被的稳定性 ${ }^{[10,45]}$ 。如受干扰严重的森林中, 具有 $\mathrm{R}$ 对策和次级 CSR 对策的植物频繁出现, 林分结构趋于简单, 生物量存储低, 从而降低了生态系统的能量耗散能力, 这可能将导致生态系统状态失调而 
损害生态系统的稳定性和生态系统服务功能的供给 ${ }^{[92]}$ 。在许多海岸带, 也出现由于水位波动加剧导致竞争 型和耐胁迫型植物减少而杂草型植物增加的现象, 对湿地生态系统过程与功能产生了不利影响 ${ }^{[93]}$ 。因此, 利 用 CSR 理论可分析预测气候变化和人为活动对植被及生态系统的潜在影响, 从而有针对性地采取适应性管 理对策。

\section{3 问题与展望}

自 1974 年 Grime 首次提出 CSR 对策的概念以来, 经过近半个世纪的发展, 已经形成了比较完整的理论和 方法体系。综合已有的研究成果, CSR 理论具有三方面的突出优势: (1) 与经济型谱和大小谱作为植物主导 功能谱的实证结果相一致, 而目前还没有其它的生态对策理论可以同时解释功能谱的这两条主轴; (2) 将植 物的生态对策与功能性状的变异空间建立联系, 因此可以利用性状的尺度灵活性,将 CSR 对策分析应用于物 种、群落和生态系统等不同水平的生态学研究; (3) CSR 对策可以作为一个通用概念, 在全球尺度上对各种实 验结果进行比较研究, 从而形成一般性结论。同时, CSR 理论为物种功能和自然选择之间提供了一个理论联 系, 暗示了在生物圈内普遍存在的选择压力, 因此可以成为普适性理论而延伸到对其它生物生态对策的研 究 ${ }^{[8]}$ 。尽管 CSR 理论已经得到很多研究的广泛验证并应用于多种尺度上的生态学研究,但仍有一些问题值 得进一步深人探讨。

\section{1 第四区缺失问题}

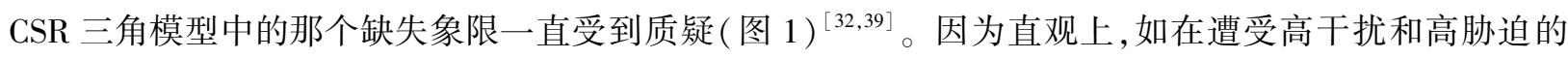
荒漠地区,仍有沙生柽柳 (Tamarix taklamakanensis) 生存 ${ }^{[94]}$ 。而本质上,第四区缺失问题是对于胁迫和干扰的 界定仍存有争议。首先, CSR 理论假设植物对胁迫或干扰具有一致性的响应特征,但事实上不同物种的响应 是不同的。如在沙漠中, 仙人掌通过肉质茎增强抗旱性, 短命植物则通过成熟植株死亡以避免干旱 ${ }^{[54]}$ 。而干 扰还存在频率和强度的不同 ${ }^{[95]}$, 并往往与胁迫交互作用 ${ }^{[96-97]}$ 。对中欧植物区系的研究发现,生境生产力与 干扰强度对物种生态位存在正交互效应,但与干扰频率的交互性不明显, 因此有很多物种可以生活在受频繁 干扰的贫㾑生境, 但很少有物种可以生活在受严重干扰的贫癐生境(支持 CSR 理论) ${ }^{[98]}$ 。 Laughlin ${ }^{[99]}$ 认为, 考虑到胁迫和干扰的多维特性, CSR 理论对于植物性状多维变异的解释可能过于简单化。事实上, 相对于生 产力-多样性关系的研究, 目前对于干扰-多样性关系以及干扰对生产力-多样性关系的影响等相关研究明显不 足 ${ }^{[100-102]}$ 。因此, 今后需要综合考虑不同生境生产力、干扰频率和强度下的植物生态对策和多样性变化。

\section{2 性状变异主轴以外的变异}

CSR 对策反映植物功能性状变异的主轴, 但不能解释所有的性状变异, 这也是 CSR 理论遭受争议的一个 方面 ${ }^{[54,103]}$ 。首先, 性状变异维度既有性状数量上的权衡, 还包括根、茎、叶、果实等多个器官性状间的权 衡 ${ }^{[04-105]}$ 。如有研究发现, 叶片、果实和种子的大小之间可能形成三角权衡关系 ${ }^{[106]}$, 根、茎、叶 3 种器官的关 键性状在资源利用上存在相互权衡和补偿 ${ }^{[107]}$, 等。但目前还不能确定根、茎、果实的性状变异是受叶性状的 直接影响, 还是各器官的性状协同变异只是因为受共同的环境因素影响, 以及各器官的性状变异能否协调一 致地反映植物整体的生态对策。再者, CSR 理论主要针对植物在定居成熟阶段的生态对策,但繁殖更新阶段 的生态对策对种群适应力和持久力具有决定意义 ${ }^{[108-109]}$ 。虽然 CSR 理论并不排除性状变异主轴外其它变异 轴的存在, 如性状变异空间第三维特化为种子权衡轴 ${ }^{[27]}$, 但目前对于共存物种的繁殖更新如何与营养生长协 同进化知之甚少 ${ }^{[110-112]}$ 。已有研究发现这些次变异轴对理解物种适应性和群落构建可能是至关重要的, 如 对于 CSR 对策相近的两种兰科植物 (Ophrys benacensis 和 Ophrys sphegodes) 需结合它们特有的繁殖特征才能进 一步解释这两个物种共存与多度差异 ${ }^{[113]}$ 。因此, 从多器官和生活史等多方面增加性状的维度, 有助于更好 地理解植物的生态对策, 也将进一步发展和完善 CSR 理论。

3.3 性状的种内变异和谱系关系

以往 CSR 对策的相关研究多基于性状的物种均值, 近年来越来越多的研究认为, 植物生态学研究中应考 
虑种内性状变异 ${ }^{[114-115]}$ 。一些功能性状的可塑性明显要比其它性状大, 如比叶面积 ${ }^{[16]}$, 这将导致同一物种

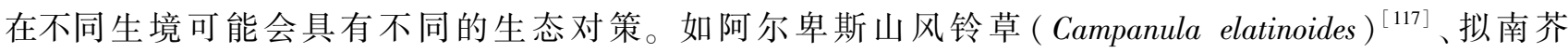
(Arabidopsis thaliana $)^{[118]}$ 、高山石竹(Dianthus pseudocrinitus) ${ }^{[119]}$ 等,都发现他们的 CSR 对策存在种内变异。 种内性状变异信息可用于评价物种生态对策的宽度和多样性 ${ }^{[17]}$, 有助于更好地理解植物对环境异质性的适 应 ${ }^{[120-121]}$ 。同时, 功能性状又是与物种系统发育史息息相关, 可以说是先天遗传因素和后天环境因素共同作 用的结果 ${ }^{[122-123]}$ 。因此在对植物适应性进化进行相关研究时, 有必要对谱系关系和环境因素进行区 别 ${ }^{[24-126]}$ 。生态对策反映影响物种适合度的功能性状间权衡, 这也为生态学和进化生物学提供了一个概念 性联系, 从而将短时期内局地尺度的生态学过程和全球尺度进化史上发生的生态学过程联系在一起, 揭示群 落物种共存和生物多样性的形成机制 ${ }^{[27-128]}$ 。因此, 今后在基于功能性状的植物生态对策研究中应该重视 种内变异和谱系关系。

\section{参考文献 (References) :}

[ 1 ] Díaz S, Kattge J, Cornelissen J H C, Wright I J, Lavorel S, Dray S, Reu B, Kleyer M, Wirth C, Prentice I C, Garnier E, Bönisch G, Westoby M, Poorter H, Reich P B, Moles A T, Dickie J, Gillison A N, Zanne A E, Chave J, Wright S J, Sheremet' ev S N, Jactel H, Baraloto C, Cerabolini B, Pierce S, Shipley B, Kirkup D, Casanoves F, Joswig J S, Günther A, Falczuk V, Rüger N, Mahecha M D, Gorné L D. The global spectrum of plant form and function. Nature, 2016, 529(7585): 167-171.

[ 2 ] Westoby M, Falster D S, Moles A T, Vesk P A, Wright I J. Plant ecological strategies: some leading dimensions of variation between species. Annual Review of Ecology and Systematics, 2002, 33: 125-159.

[ 3 ] Pierce S, Negreiros D, Cerabolini B E L, Kattge J, Díaz S, Kleyer M, Shipley B, Wright S J, Soudzilovskaia N A, Onipchenko V G, van Bodegom P M, Frenette-Dussault C, Weiher E, Pinho B X, Cornelissen J H C, Grime J P, Thompson K, Hunt R, Wilson P J, Buffa G, Nyakunga O C, Reich P B, Caccianiga M, Mangili F, Ceriani R M, Luzzaro A, Brusa G, Siefert A, Barbosa N P U, Chapin III F S, Cornwell W K, Fang J Y, Fernandes G W, Garnier E, Stradic S L, Peñuelas J, Melo F P L, Slaviero A, Tabarelli M, Tampucci D. A global method for calculating plant CSR ecological strategies applied across biomes world-wide. Functional Ecology, 2017, 31(2) : 444-457.

[ 4 ] Díaz S, Cabido M. Plant functional types and ecosystem function in relation to global change. Journal of Vegetation Science, 1997, 8(4) : 463-474.

[ 5 ] Grime J P. Plant Strategies and Vegetation Processes. New York: John Wiley \& Sons, 1979.

[ 6 ] Guo W Y, van Kleunen M, Winter M, Weigelt P, Stein A, Pierce S, Pergl J, Moser D, Maurel N, Lenzner B, Kreft H, Essl F, Dawson W, Pyšek P. The role of adaptive strategies in plant naturalization. Ecology Letters, 2018, 21(9): 1380-1389.

[ 7 ] Reich P B, Wright I J, Cavender-Bares J, Craine J M, Oleksyn J, Westoby M, Walters M B. The evolution of plant functional variation: traits, spectra, and strategies. International Journal of Plant Science, 2003, 164(S3) : S143-S164.

[ 8 ] Grime J P, Pierce S. The Evolutionary Strategies that Shape Ecosystems. New Jersey: Wiley-Blackwell, 2012.

[ 9 ] Grime J P. Vegetation classification by reference to strategies. Nature, 1974, 250(5461) : 26-31.

[10] Grime J P. Plant Strategies, Vegetation Processes, and Ecosystem Properties. England: John Wiley \& Sons, 2001.

[11] Hodgson J G, Wilson P J, Hunt R, Grime J P, Thompson K. Allocating C-S-R plant functional types: a soft approach to a hard problem. Oikos, $1999,85(2): 282-294$.

[12] Grime J P. Evidence for the existence of three primary strategies in plants and its relevance to ecological and evolutionary theory. The American Naturalist, 1977, 111(982): 1169-1194.

[13 ] Grime J P, Thompson K, Hunt R, Hodgson J G, Cornelissen J H C, Rorison I H, Hendry G A F, Ashenden T W, Askew A P, Band S R, Booth R E, Bossard C C, Campbell B D, Cooper J E L, Davison A W, Gupta P L, Hall W, Hand D W, Hannah M A, Hillier S H, Hodkinson D J, Jalili A, Liu Z, Mackey J M L, Matthews N, Mowforth M A, Neal A M, Reader R J, Reiling K, Ross-Fraser W, Spencer R E, Sutton F, Tasker D E, Thorpe P C, Whitehouse J. Integrated screening validates primary axes of specialisation in plants. Oikos, 1997, 79(2) : 259-281.

[14] Grime J P, Hodgson J G, Hunt R. Comparative Plant Ecology : A Functional Approach to Common British Species. London: Unwin Hyman, 1988.

[15] Cerabolini B E L, Brusa G, Ceriani R M, De Andreis R, Luzzaro A, Pierce S. Can CSR classification be generally applied outside Britain? Plant Ecology, 2010, 210(2): 253-261.

[16] Caccianiga M, Luzzaro A, Pierce S, Ceriani R M, Cerabolini B. The functional basis of a primary succession resolved by CSR classification. Oikos, 2006, 112(1): 10-20.

[17] Pierce S, Brusa G, Vagge I, Cerabolini B E L. Allocating CSR plant functional types: the use of leaf economics and size traits to classify woody and herbaceous vascular plants. Functional Ecology, 2013, 27(4) : 1002-1010.

[18] Li Y Z, Shipley B. An experimental test of CSR theory using a globally calibrated ordination method. PLoS One, 2017, $12(4)$ : e0175404.

[19] Hunt R, Hodgson J G, Thompson K, Bungener P, Dunnett N P, Askew A P. A new practical tool for deriving a functional signature for herbaceous 
vegetation. Applied Vegetation Science, 2004, 7(2): 163-170.

[20] Bornhofen S, Barot S, Lattaud C. The evolution of CSR life-history strategies in a plant model with explicit physiology and architecture. Ecological Modelling, 2011, 222(1): 1-10.

[21] Laughlin D C, Leppert J J, Moore M M, Sieg C H. A multi-trait test of the leaf-height-seed plant strategy scheme with 133 species from a pine forest flora. Functional Ecology, 2010, 24(3): 493-501.

[22] Douma J C, Aerts R, Witte J P M, Bekker R M, Kunzmann D, Metselaar K, van Bodegom P M. A combination of functionally different plant traits provides a means to quantitatively predict a broad range of species assemblages in NW Europe. Ecography, 2012, 35(4) : 364-373.

[23] Pierce S, Brusa G, Sartori M, Cerabolini B E L. Combined use of leaf size and economics traits allows direct comparison of hydrophyte and terrestrial herbaceous adaptive strategies. Annals of Botany, 2012, 109(5) : 1047-1053.

[24] Hodgson J G, Santini B A, Marti G M, Pla F R, Jones G, Bogaard A, Charles M, Font X, Ater M, Taleb A, Poschlod P, Hmimsa Y, Palmer C, Wilson P J, Band S R, Styring A, Diffey C, Green L, Nitsch E, Stroud, E, Romo-Díez A, de Torres Espuny L, Warham G. Trade-offs between seed and leaf size (seed-phytomer-leaf theory) : functional glue linking regenerative with life history strategies $\cdots$ and taxonomy with ecology? Annals of Botany, 2017, 120(5): 633-652.

[25] Díaz S, Hodgson J G, Thompson K, Cabido M, Cornelissen J H C, Jalili A, Montserrat-Martí G, Grime J P, Zarrinkamar F, Asri Y, Band S R, Basconcelo S, Castro-Díez P, Funes G, Hamzehee B, Khoshnevi M, Pérez-Harguindeguy N, Pérez-Rontomé M C, Shirvany F A, Vendramini F, Yazdani S, Abbas-Azimi R, Bogaard A, Boustani S, Charles M, Dehghan M, de Torres-Espuny L, Falczuk V, Guerrero-Campo J, Hynd A, Jones G, Kowsary E, Kazemi-Saeed F, Maestro-Martínez M, Romo-Díez A, Shaw S, Siavash B, Villar-Salvador P, Zak M R. The plant traits that drive ecosystems: evidence from three continents. Journal of Vegetation Science, 2004, 15(3) : 295-304.

[26] Freschet G T, Cornelissen J H C, van Logtestijn R S P, Aerts R. Evidence of the 'plant economics spectrum' in a subarctic flora. Journal of Ecology, 2010, 98(2): 362-373.

[27] Pierce S, Bottinelli A, Bassani I, Ceriani R M, Cerabolini B E L. How well do seed production traits correlate with leaf traits, whole-plant traits and plant ecological strategies? Plant Ecology, 2014, 215(11): 1351-1359.

[28] 刘志民, 赵晓英, 范世香. Grime 的植物对策思想和生态学研究理念. 地球科学进展, 2003, 18(4): 603-608.

[29] Reich P B. The world-wide 'fast-slow' plant economics spectrum: a traits manifesto. Journal of Ecology, 2014, 102(2) : 275-301.

[30] Silva J L A, Souza A F, Caliman A, Voigt E L, Lichston J E. Weak whole-plant trait coordination in a seasonally dry South American stressful environment. Ecology and Evolution, 2018, 8(1): 4-12.

[31] de Paula L F A, Negreiros D, Azevedo L O, Fernandes R L, Stehmann J R, Silveira F A O. Functional ecology as a missing link for conservation of a resource-limited flora in the Atlantic forest. Biodiversity and Conservation, 2015, 24(9) : 2239-2253.

[32] Craine J M. Plant strategy theories: replies to Grime and Tilman. Journal of Ecology, 2007, 95(2): 235-240.

[33] Negreiros D, Le Stradic S, Fernandes G W, Rennó H C. CSR analysis of plant functional types in highly diverse tropical grasslands of harsh environments. Plant Ecology, 2014, 215(4) : 379-388.

[34] Zelnik I, Čarni A. Distribution of plant communities, ecological strategy types and diversity along a moisture gradient. Community Ecology, 2008, 9 (1) : 1-9.

[35] 王斌世, 张荣. 半干旱区农田杂草的生活史对策研究. 草业学报, 2011, 20(1): 257-260.

[36] Rosenfield M F, Müller S C, Overbeck G E. Short gradient, but distinct plant strategies: the CSR scheme applied to subtropical forests. Journal of Vegetation Science, 2019, 30(5) : 984-993.

[37] Catorci A, Vitanzi A, Tardella F M. Variations in CSR strategies along stress gradients in the herb layer of submediterranean forests (central Italy). Plant Ecology and Evolution, 2011, 144(3): 299-306.

[38] Elmas E. Ecophysiological characteristics and CSR strategies of four dune species. Annales Botanici Fennici, 2017, 54(4/6): 353-360.

[39] Westoby M. A leaf-height-seed (LHS) plant ecology strategy scheme. Plant and Soil, 1998, 199(2) : 213-227.

[40] Frenette-Dussault C, Shipley B, Léger J F, Meziane D, Hingrat Y. Functional structure of an arid steppe plant community reveals similarities with Grime's $C$-S-R theory. Journal of Vegetation Science, 2012, 23(2) : 208-222.

[41] Pierce S, Luzzaro A, Caccianiga M, Ceriani R M, Cerabolini B. Disturbance is the principal $\alpha$-scale filter determining niche differentiation, coexistence and biodiversity in an alpine community. Journal of Ecology, 2007, 95(4) : 698-706.

[42] Cerabolini B, Pierce S, Luzzaro A, Ossola A. Species evenness affects ecosystem processes in situ via diversity in the adaptive strategies of dominant species. Plant Ecology, 2010, 207(2) : 333-345.

[43] Moog D, Kahmen S, Poschlod P. Application of CSR-and LHS-strategies for the distinction of differently managed grasslands. Basic and Applied Ecology, 2005, 6(2): 133-143.

[44] Colasanti R L, Hunt R, Askew A P. A self-assembling model of resource dynamics and plant growth incorporating plant functional types. Functional Ecology , 2001, 15(5): 676-687.

[45] Wonkka C L, Lafon C W, Hutton C M, Joslin A J. A CSR classification of tree life history strategies and implications for ice storm damage. Oikos, $2013,122(2): 209-222$.

[46] Kopecký M, Hédl R, Szabó P. Non-random extinctions dominate plant community changes in abandoned coppices. Journal of Applied Ecology, $2013,50(1): 79-87$. 
[47] Cross E L, Green P T, Morgan J W. A plant strategy approach to understand multidecadal change in community assembly processes in Australian grassy woodlands. Journal of Ecology, 2015, 103(5) : 1300-1307.

[48] Timmermann A, Damgaard C, Strandberg M T, Svenning J C. Pervasive early 21st-century vegetation changes across Danish semi-natural ecosystems: more losers than winners and a shift towards competitive, tall-growing species. Journal of Applied Ecology, 2015, 52(1) : 21-30.

[49] Boatman N D, Jones N E, Conyers S T, Pietravalle S. Development of plant communities on set-aside in England. Agriculture, Ecosystems \& Environment, 2011, 143(1): 8-19.

[50] Vicente J R, Pinto A T, Araújo M B, Verburg P H, Lomba A, Randin C F, Guisan A, Honrado J P. Using Life Strategies to Explore the Vulnerability of Ecosystem Services to Invasion by Alien Plants. Ecosystems, 2013, 16(4) : 678-693.

[51] Fratte M D, Bolpagni R, Brusa G, Caccianiga M, Pierce S, Zanzottera M, Cerabolini B E L. Alien plant species invade by occupying similar functional spaces to native species. Flora, 2019, 257: 151419.

[52] Bakker J, Wilson S. Competitive abilities of introduced and native grasses. Plant Ecology, 2001, 157(2): 119-127.

[53] Vuković N, Miletić M, Milović M, Jelaska S D. Grime's CSR strategies of the invasive plants in Croatia. Periodicum Biologorum, 2014, 116( 3): 323-329.

[54] Wilson J B, Lee W G. C-S-R triangle theory: community-level predictions, tests, evaluation of criticisms, and relation to other theories. Oikos, $2000,91(1): 77-96$.

[55] 许驭丹, 董世鬼, 李帅, 沈豪. 植物群落构建的生态过滤机制研究进展. 生态学报, 2019, 39(7): 2267-2281.

[56] Kraft N J B, Adler P B, Godoy O, James E C, Fuller S, Levine J M. Community assembly, coexistence and the environmental filtering metaphor. Functional Ecology, 2015, 29(5): 592-599.

[57] Stokes C J, Archer S R. Niche differentiation and neutral theory: an integrated perspective on shrub assemblages in a parkland savanna. Ecology, 2010, 91(4): 1152-1162.

[58] Wennekes P L, Rosindell J, Etienne R S. The neutral-niche debate: a philosophical perspective. Acta Biotheoretica, 2012 , 60(3) : $257-271$.

[59］柴永福, 岳明. 植物群落构建机制研究进展. 生态学报, 2016, 36( 15): 4557-4572.

[60] Mason N W H, de Bello F, Doležal J, Lepš J. Niche overlap reveals the effects of competition, disturbance and contrasting assembly processes in experimental grassland communities. Journal of Ecology, 2011, 99(3) : 788-796.

[61] Maire V, Gross N, Börger L, Proulx R, Wirth C, Pontes L D S, Soussana J F, Louault F. Habitat filtering and niche differentiation jointly explain species relative abundance within grassland communities along fertility and disturbance gradients. New Phytologist, 2012, 196(2) : 497-509.

[62] Spasojevic M J, Suding K N. Inferring community assembly mechanisms from functional diversity patterns: the importance of multiple assembly processes. Journal of Ecology, 2012, 100(3) : 652-661.

[63] Bernard-Verdier M, Navas M L, Vellend M, Violle C, Fayolle A, Garnier E. Community assembly along a soil depth gradient: contrasting patterns of plant trait convergence and divergence in a Mediterranean rangeland. Journal of Ecology, 2012, 100( 6) : 1422-1433.

[64] Verheijen L M, Aerts R, Bönisch G, Kattge J, Van Bodegom P M. Variation in trait trade-offs allows differentiation among predefined plant functional types: implications for predictive ecology. New Phytologist, 2016, 209(2) : 563-575.

[65] Rosado B H P, de Mattos E A. On the relative importance of CSR ecological strategies and integrative traits to explain species dominance at local scales. Functional Ecology, 2017, 31(10): 1969-1974.

[66] Grime J P. Trait convergence and trait divergence in herbaceous plant communities: mechanisms and consequences. Journal of Vegetation Science, 2006, 17(2): 255-260.

[67] Cerabolini B E L, Pierce S, Verginella A, Brusa G, Ceriani R M, Armiraglio S. Why are many anthropogenic agroecosystems particularly speciesrich? Plant Biosystems-An International Journal Dealing with all Aspects of Plant Biology, 2016, 150(3) : $550-557$.

[68] Chase J M. Community assembly: when should history matter? Oecologia, 2003, 136(4): 489-498.

[69] Purschke O, Schmid B C, Sykes M T, Poschlod P, Michalski S G, Durka W, Kühn I, Winter M, Prentice H C. Contrasting changes in taxonomic, phylogenetic and functional diversity during a long-term succession: insights into assembly processes. Journal of Ecology, 2013, 101 (4) : 857-866

[70] Li Y Z, Shipley B. Community divergence and convergence along experimental gradients of stress and disturbance. Ecology, 2018, 99(4): $775-781$.

[71] Barba-Escoto L, Ponce-Mendoza A, García-Romero A, Calvillo-Medina R P. Plant community strategies responses to recent eruptions of Popocatépetl volcano, Mexico. Journal of Vegetation Science, 2019, 30(2) : 375-385.

[72] Kelemen A, Tóthmérész B, Valkó O, Miglécz T, Deák B, Török P. New aspects of grassland recovery in old-fields revealed by trait-based analyses of perennial-crop-mediated succession. Ecology and Evolution, 2017, 7(7) : 2432-2440.

[73] Prévosto B, Kuiters L, Bernhardt-Römermann M, Dölle M, Schmidt W, Hoffmann M, Van Uytvanck J, Bohner A, Kreiner D, Stadler J, Klotz S, Brandl R. Impacts of land abandonment on vegetation: successional pathways in european habitats. Folia Geobotanica, 2011, 46(4) : 303-325.

[74] Pywell R F, Bullock J M, Roy D B, Warman L, Walker K J, Rothery P. Plant traits as predictors of performance in ecological restoration. Journal of Applied Ecology, 2003, 40(1): 65-77.

[75] 郑颖, 温仲明, 宋光, 丁曼. 延河流域森林草原区不同植物功能型适应策略及功能型物种数量随退耕年限的变化. 生态学报, 2015 , 35 (17) : 5834-5845. 
[76] Paušič A, Čarni A. Functional Response traits and plant community strategy indicate the stage of secondary succession. Hacquetia, 2012, 11(2) : 209-225.

[77] Mori A S, Cornelissen J H C, Fujii S, Okada K I, Isbell F. A meta-analysis on decomposition quantifies afterlife effects of plant diversity as a global change driver. Nature Communications, 2020, 11(1): 4547.

[78] Freschet G T, Aerts R, Cornelissen J H C. A plant economics spectrum of litter decomposability. Functional Ecology, 2012, 26( 1) : 56-65.

[79] Handa I T, Aerts R, Berendse F, Berg M P, Bruder A, Butenschoen O, Chauvet E, Gessner M O, Jabiol J, Makkonen M, McKie B G, Malmqvist B, Peeters E T H M, Scheu S, Schmid B, van Ruijven J, Vos V C A, Hättenschwiler S. Consequences of biodiversity loss for litter decomposition across biomes. Nature, 2014, 509(7499) : 218-221.

[80] Cornwell W K, Cornelissen J H C, Amatangelo K, Dorrepaal E, Eviner V T, Godoy O, Hobbie S E, Hoorens B, Kurokawa H, PérezHarguindeguy N, Quested H M, Santiago L S, Wardle D A, Wright I J, Aerts R, Allison S D, Van Bodegom P, Brovkin V, Chatain A, Callaghan T V, Díaz S, Garnier E, Gurvich D E, Kazakou E, Klein J A, Read J, Reich P B, Soudzilovskaia N A, Vaieretti M V, Westoby M. Plant species traits are the predominant control on litter decomposition rates within biomes worldwide. Ecology Letters, 2008, 11( 10): 1065-1071.

[81] Grasset C, Delolme C, Arthaud F, Bornette G. Carbon allocation in aquatic plants with contrasting strategies: the role of habitat nutrient content. Journal of Vegetation Science, 2015, 26( 5): 946-955.

[82] Zhang F Y, Quan Q, Song B, Sun J, Chen Y J, Zhou Q P, Niu S L. Net primary productivity and its partitioning in response to precipitation gradient in an alpine meadow. Scientific Reports, 2017, 7(1): 15193.

[83] Jenkins D G, Pierce S. General allometric scaling of net primary production agrees with plant adaptive strategy theory and has tipping points. Journal of Ecology, 2017, 105(4): 1094-1104.

[84] Gillman L N, Wright S D. The influence of productivity on the species richness of plants: a critical assessment. Ecology, 2006, 87 ( 5 ) : 1234-1243.

[85] Hooper D U, Chapin III F S, Ewel J J, Hector A, Inchausti P, Lavorel S, Lawton J H, Lodge D M, Loreau M, Naeem S, Schmid B, Setälä H, Symstad A J, Vandermeer J, Wardle D A. Effects of biodiversity on ecosystem functioning: a consensus of current knowledge. Ecological Monographs, 2005, 75(1): 3-35.

[86] 赵峰侠, 徐明. 生产力与生物多样性关系的研究进展. 自然资源学报, 2018, 33(11): 2046-2056.

[87] Grime J P. Competitive exclusion in herbaceous vegetation. Nature, 1973, 242(5396) : 344-347.

[88] Kelemen A, Török P, Valkó O, Miglécz T, Tóthmérész B. Mechanisms shaping plant biomass and species richness : plant strategies and litter effect in alkali and loess grasslands. Journal of Vegetation Science, 2013, 24(6) : 1195-1203.

[89] Zhang H Y, Wu J G. A statistical thermodynamic model of the organizational order of vegetation. Ecological Modelling, 2002, 153(1/2) : 69-80.

[90] Lin H, Cao M, Stoy P C, Zhang Y P. Assessing self-organisation of plant communities - A thermodynamic approach. Ecological Modelling, 2009, $220(6): 784-790$.

[91] Norris C, Hobson P, Ibisch P L. Microclimate and vegetation function as indicators of forest thermodynamic efficiency. Journal of Applied Ecology, $2012,49(3): 562-570$.

[92] Clewell A F, Aronson J. Motivations for the restoration of ecosystems. Conservation Biology, 2006, 20(2) : $420-428$.

[93] Abrahams C. Climate change and lakeshore conservation: a model and review of management techniques. Hydrobiologia, 2008,613 ( 1) : 33-43.

[94] He X D, Gao Y B, Ren A Z. Role of wind-sand disturbance in the formation and development of Tamarix taklamakanensis community. Acta Botanica Sinica, 2003, 45(11): 1285-1290.

[95] Herben T, Klimešová J, Chytrý M. Effects of disturbance frequency and severity on plant traits: an assessment across a temperate flora. Functional Ecology, 2018, 32(3): 799-808.

[96] Sonnier G, Shipley B, Navas M L. Quantifying relationships between traits and explicitly measured gradients of stress and disturbance in early successional plant communities. Journal of Vegetation Science, 2010, 21(6) : 1014-1024.

[97] Wang J, Zhang C Y, Yang H, Mou C X, Mo L, Luo P. Plant community ecological strategy assembly response to yak grazing in an alpine meadow on the eastern Tibetan Plateau. Land Degradation \& Development, 2018, 29(9) : 2920-2931.

[98] Herben T, Klimešová J, Chytrý M. Philip Grime's fourth corner: are there plant species adapted to high disturbance and low productivity? Oikos, $2018,127(8): 1125-1131$.

[99] Laughlin D C. The intrinsic dimensionality of plant traits and its relevance to community assembly. Journal of Ecology, 2014 , 102 ( 1) : $186-193$.

[100] Miller A D, Roxburgh S H, Shea K. How frequency and intensity shape diversity-disturbance relationships. Proceedings of the National Academy of Sciences of the United States of America, 2011, 108(14) : 5643-5648.

[101] 刘志民, 陈怀顺, 刘新民. 干扰与植被关系研究的特点和面临的挑战. 地球科学进展, 2002, 17(4): 582-587.

[102] Huston M A. Disturbance, productivity, and species diversity: empiricism vs. logic in ecological theory. Ecology, 2014, 95(9): 2382-2396.

[103] Walker A P, McCormack M L, Messier J, Myers-Smith I H, Wullschleger S D. Trait covariance: the functional warp of plant diversity? New Phytologist, 2017, 216(4): 976-980.

[104] Westoby M, Wright I J. The leaf size-twig size spectrum and its relationship to other important spectra of variation among species. Oecologia, $2003,135(4): 621-628$.

[105] Kleyer M, Minden V. Why functional ecology should consider all plant organs: an allocation- based perspective. Basic and Applied Ecology, 
$2015,16(1): 1-9$

[106] Cornelissen J H C. A triangular relationship between leaf size and seed size among woody species: allometry, ontogeny, ecology and taxonomy. Oecologia, 1999, 118(2): 248-255

[107] Fortunel C, Fine P V A, Baraloto C. Leaf, stem and root tissue strategies across 758 neotropical tree species. Functional Ecology, 2012, 26(5): 1153-1161.

[108] Eriksson O, Jakobsson A. Recruitment trade-offs and the evolution of dispersal mechanisms in plants. Evolutionary Ecology, 1999, 13(4): 411-423.

[109] Salguero-Gómez R. Applications of the fast-slow continuum and reproductive strategy framework of plant life histories. New Phytologist, 2017, 213 (4) : 1618-1624.

[110] Dayrell R L C, Arruda A J, Pierce S, Negreiros D, Meyer P B, Lambers H, Silveira F A O. Ontogenetic shifts in plant ecological strategies. Functional Ecology, 2018, 32(12): 2730-2741.

[111] Moles A T, Westoby M. Seed size and plant strategy across the whole life cycle. Oikos, 2006, 113(1) : 91- 105.

[112] Rüger N, Comita L S, Condit R, Purves D, Rosenbaum B, Visser M D, Wright S J, Wirth C. Beyond the fast-slow continuum: demographic dimensions structuring a tropical tree community. Ecology Letters, 2018, 21(7): 1075-1084.

[113] Pierce S, Vagge I, Brusa G, Cerabolini B E L. The intimacy between sexual traits and Grime's CSR strategies for orchids coexisting in seminatural calcareous grassland at the Olive Lawn. Plant Ecology, 2014, 215(5): 495-505.

[114] Albert C H, de Bello F, Boulangeat I, Pellet G, Lavorel S, Thuiller W. On the importance of intraspecific variability for the quantification of functional diversity. Oikos, 2012, 121(1): 116-126.

[115] Tautenhahn S, Grün-Wenzel C, Jung M, Higgins S, Römermann C. On the relevance of intraspecific trait variability - a synthesis of 56 dry grassland sites across Europe. Flora, 2019, 254: 161- 172.

[116] Shipley B. Plasticity in relative growth rate and its components following a change in irradiance. Plant, Cell \& Environment, 2000, 23(11): 1207-1216.

[117] Giupponi L. Intraspecific variation in functional strategy and leaf shape of Campanula elatinoides reveals adaptation to climate. Flora, 2020, 268: 151605 .

[118] May R L, Warner S, Wingler A. Classification of intra-specific variation in plant functional strategies reveals adaptation to climate. Annals of Botany, 2017, 119(8) : 1343-1352.

[119] Behroozian M, Ejtehadi H, Memariani F, Pierce S, Mesdaghi M. Are endemic species necessarily ecological specialists? Functional variability and niche differentiation of two threatened Dianthus species in the montane steppes of northeastern Iran. Scientific Reports, 2020, 10(1): 11774.

[120] Jung V, Violle C, Mondy C, Hoffmann L, Muller S. Intraspecific variability and trait-based community assembly. Journal of Ecology, 2010, 98 (5) : 1134-1140.

[121] Astuti G, Ciccarelli D, Roma-Marzio F, Trinco A, Peruzzi L. Narrow endemic species Bellevalia webbiana shows significant intraspecific variation in tertiary CSR strategy. Plant Biosystems-An International Journal Dealing with all Aspects of Plant Biology, 2019, 153(1): 12-18.

[122] 刘晓娟, 马克平. 植物功能性状研究进展. 中国科学: 生命科学, 2015, 45(4): 325-339.

[123] Rosado B H P, Matos I S, de A Amorim T. A matter of scale and traits: a comment on 'On the need for phylogenetic “corrections” in functional trait-based approaches' by de Bello et al. (2015). Folia Geobotanica, 2016, 51(4) : 383-387.

[124] 曹科, 饶米德, 余建中, 刘晓娟, 米湘成, 陈建华. 古田山木本植物功能性状的系统发育信号及其对群落结构的影响. 生物多样性, 2013, 21(5): 564-571.

[125 ] de Bello F, Berg M P, Dias A T C, Diniz-Filho J A F, Götzenberger L, Hortal J, Ladle R J, Lepš J. On the need for phylogenetic 'corrections' in functional trait-based approaches. Folia Geobotanica, 2015, 50(4) : 349-357.

[126] 黄建雄, 郑凤英, 米湘成. 不同尺度上环境因子对常绿阔叶林群落的谱系结构的影响. 植物生态学报, 2010, 34(3): 309-315.

[127] Munoz F, Violle C, Cheptou P O. CSR ecological strategies and plant mating systems: outcrossing increases with competitiveness but stresstolerance is related to mixed mating. Oikos, 2016, 125(9): 1296-1303.

[ 128] Cornwell W K, Westoby M, Falster D S, FitzJohn R G, O'Meara B C, Pennell M W, McGlinn D J, Eastman J M, Moles A T, Reich P B, Tank D C, Wright I J, Aarssen L, Beaulieu J M, Kooyman R M, Leishman M R, Miller E T, Niinemets Ü, Oleksyn J, Ordonez A, Royer D L, Smith S A, Stevens P F, Warman L, Wilf P, Zanne A E. Functional distinctiveness of major plant lineages. Journal of Ecology, 2014, 102(2) : 345-356. 